Research Article

\title{
Resonance between the Representation Function and Exponential Functions over Arithemetic Progression
}

\author{
Li Ma $\mathbb{D}$ and Xiaofei Yan \\ School of Mathematics and Statistics, Shandong Normal University, Jinan, Shandong 250100, China \\ Correspondence should be addressed to Xiaofei Yan; xfyan.sdu@gmail.com
}

Received 14 December 2020; Accepted 16 January 2021; Published 2 February 2021

Academic Editor: Tianping Zhang

Copyright (c) $2021 \mathrm{Li} \mathrm{Ma}$ and Xiaofei Yan. This is an open access article distributed under the Creative Commons Attribution License, which permits unrestricted use, distribution, and reproduction in any medium, provided the original work is properly cited.

Let $r(n)$ denote the number of representations of a positive integer $n$ as a sum of two squares, i.e., $n=x_{1}^{2}+x_{2}^{2}$, where $x_{1}$ and $x_{2}$ are integers. We study the behavior of the exponential sum twisted by $r(n)$ over the arithmetic progressions $\sum_{\substack{n \sim X \\ n=l \bmod q}} r(n) e\left(\alpha n^{\beta}\right)$, where $0 \neq \alpha \in \mathbb{R}, 0<\beta<1, e(x)=e^{2 \pi i x}$, and $n \sim X$ means $X<n \leq 2 X$. Here, $X>1$ is a large parameter, $1 \leq l \leq q$ are integers, and $(l, q)=1$. We obtain the upper bounds in different situations.

\section{Introduction}

In analytic number theory, the problems concerning nonlinear exponential twisting arithmetic functions arise naturally in investigating equidistribution theory, zerodistribution of $L$-functions, and so on. Let $a_{n}$ be some arithmetic number-theoretic function. We usually consider the general nonlinear exponential sum of the form

$$
S(X, \alpha)=\sum_{n \sim X} a_{n} e\left(\alpha n^{\beta}\right), \quad 0 \neq \alpha \in \mathbb{R}, 0 \leq \beta \leq 1 .
$$

Here, $n \sim X$ means $X \leq n \leq 2 X$, and $e(z)=e^{2 \pi i z}$. When $\beta=1 / 2$ and $a_{n}=\Lambda(n)$ is the von Mangoldt function, the $\operatorname{sum} S(X, \alpha)$ was studied by Vinogradov [1]. For $a_{n}=\Lambda(n)$ and $a_{n}=\mu(n)$ ( $\mu$ is the Möbius function), the sums $S(X, \alpha)$ were studied by Iwaniec, Luo, and Sarnak, and they showed these sums are intimately related to $L$-functions of $G L_{2}$. If $f$ is a holomorphic cusp form of even weight on the upper half plane, they also proved that a good upper bound of $S(X, \alpha)$ implies a quasi-Riemann hypothesis for $L(s, f)$ [2]. In addition, studying the behavior of the Fourier coefficients of automorphic forms has great significance in modern number theory. Analytic number theorists always estimate the mean value or the twisted sums such as $S(X, \alpha)$ mentioned above to obtain some information about the Fourier coefficients (for examples, see $[3-10])$.

If $\beta$ is a variable and $a_{n}$ are the Fourier coefficients of automorphic forms, these sums were studied by Ren and Ye [7] and Sun and $\mathrm{Wu}$ [11]. They proved that the resonance phenomenon occurs only when $\beta=1 / 2$ and $|\alpha|$ is close to $2 \sqrt{k}, k \in \mathbb{Z}^{+}$. Let $r(n)$ denote the number of representations of a positive integer $n$ as a sum of two squares, i.e., $n=x_{1}^{2}+x_{2}^{2}$, where $x_{1}$ and $x_{2}$ are integers. Sun and $\mathrm{Wu}$ [11] also studied the case that $a_{n}=r(n)$ and obtained the resonance phenomenon. Yan [12] studied the nonlinear exponential sum twisting the Fourier coefficients of Maass forms over the arithmetic progress and obtained an asymptotic formula for the sum

$$
\sum_{\substack{n \sim X \\ n \equiv l \bmod q}} \lambda_{g}(n) e\left(\alpha n^{\beta}\right), \quad 0 \neq \alpha \in \mathbb{R}, 0 \leq \beta \leq 1,
$$

where $g$ is a Maass cusp form for $S L(2, Z)$ and $\lambda_{g}(n)$ is the $n$ th Fourier coefficient of $g$. These analogues to the arithmetic progression are the main motivation of this paper. 
In this paper, we study the nonlinear exponential sum

$$
\sum_{\substack{n \sim X \\ n=l \bmod q}} r(n) e\left(\alpha n^{\beta}\right)
$$

where $0 \neq \alpha \in \mathbb{R}, 0<\beta<1$. Here, $X>1$ is a large parameter. $1 \leq l \leq q$ are integers, and $(l, q)=1$. We consider the case that $q$ tends to infinity as $X \longrightarrow \infty$ and obtain analogues of the result of Sun and $\mathrm{Wu}[11]$. result.

The principal aim of this paper is to prove the following

Theorem 1. Let $X>1,0<\beta<1$, and $0 \neq \alpha \in \mathbb{R}$. Let $l, q \in \mathbb{N}$ and $l \leq q \leq X^{1 / 2}$. Let $\delta=(q, 4)$.

(i) If $|\alpha| \beta q X^{\beta}<(1 / 4) \sqrt{\delta X}$, then we have

$$
\sum_{\substack{n \sim X \\ n \equiv l \bmod q}} r(n) e\left(\alpha n^{\beta}\right) \ll q^{(1 / 3)+\varepsilon} X\left(|\alpha| \beta X^{\beta}\right)^{-1}
$$

(ii) If $|\alpha| \beta q X^{\beta} \geq(1 / 4) \sqrt{\delta X}$ and $\beta \neq(1 / 2)$, then we have

$$
\sum_{\substack{n \sim X \\ n \equiv l \operatorname{lmod} q}} r(n) e\left(\alpha n^{\beta}\right) \ll q^{(1 / 2)+\varepsilon}|2 \beta-1|^{-(1 / 2)}\left(|\alpha| \beta X^{\beta}\right)^{1+\varepsilon} .
$$

(iii) If $|\alpha| \beta q X^{\beta} \geq(1 / 4) \sqrt{\delta X}$ and $\beta=1 / 2$, then for $|\alpha|<1 /$ or $|\alpha|>\sqrt{X} / q$, we have

$$
\sum_{\substack{n \sim X \\ n \equiv l \bmod q}} r(n) e\left(\alpha n^{\beta}\right) \ll q^{(1 / 2)+\varepsilon} X^{(1 / 4)+\varepsilon}|\alpha|^{(1 / 2)+\varepsilon} .
$$

For $(1 / q) \leq|\alpha| \leq(\sqrt{X} / q)$, we have

$$
\begin{aligned}
\sum_{\substack{n \sim X \\
n \equiv l \operatorname{lmod} q}} r(n) e\left(\alpha n^{\beta}\right)= & \frac{1}{q} \sum_{c \mid q} \varepsilon\left(a, n_{c}\right) c^{-3 / 2} X^{3 / 4} r\left(n_{c}, Q^{*}\right)\left|S\left(-l,-\overline{D_{c}} n_{c} ; c\right)\right| n_{c}^{-1 / 4} G_{Q}(c, a) \\
& +O\left(q^{(1 / 2)+\varepsilon} X^{(1 / 4)+\varepsilon}|\alpha|^{(1 / 2)+\varepsilon}\right),
\end{aligned}
$$

where

$$
\varepsilon\left(a, n_{c}\right)=\frac{\delta_{c} \varepsilon_{\alpha} a_{1}}{2} \int_{1}^{2} u^{-1 / 4} e\left(\operatorname{sgn}(\alpha)\left(|\alpha|-\frac{2 \sqrt{\delta n_{c}}}{2 c}\right) \sqrt{X u}\right) \mathrm{d} u
$$

and $\delta_{c}=1$ or 0 according to if there exists a positive integer $n_{c}$ for $c \mid q$ satisfying

$$
|c| \alpha\left|-\sqrt{\delta n_{c}}\right| \leq X^{-1 / 2}
$$

or not.

(iv) In particular, if $|\alpha|=(\sqrt{\delta k} / q)$ with $1 \leq k \leq(X / \delta)$, then we have

$$
\begin{aligned}
\sum_{\substack{n \sim X \\
n \equiv l \bmod q}} r(n) e\left( \pm \frac{\sqrt{\delta k n}}{q}\right)= & \frac{2 \sqrt{2}}{3}\left(2^{3 / 4}-1\right) \delta^{-1 / 4}(1 \pm i)\left|S\left(-l,-\overline{D_{c}} k ; q\right)\right| q^{-5 / 2} X^{3 / 4} r\left(k, Q^{*}\right) k^{-1 / 4} G_{Q}(q, a) \\
& +O\left(k^{-1 / 4} X^{1 / 4}(q X k)^{\varepsilon}\right) .
\end{aligned}
$$

To prove Theorem 1, we shall follow the steps in $[7,11,12]$ first. Then, we will use a new Voronoi-type summation formula generalized by $\mathrm{Hu}$ et al. [13] to get the asymptotic formula, and this is the key to success. Thus, we can get the Kloosterman sum, use Weil's bound to get the saving in the $q$-aspect, and then obtain a similar main term as that in [12].

\section{Some Lemmas}

To prove Theorem 1, we need to quote some lemmas. First, we consider the Kloosterman sum, which is defined as

$$
S(m, n ; c)=\sum_{d \bmod c} * e\left(\frac{m d+n \bar{d}}{c}\right)
$$


where $\bar{d}$ denotes the inverse of $d$ modulo $c$. The famous Weil's bound of the Kloosterman sum is

$$
|S(m, n ; c)| \leq(m, n, c)^{1 / 2} c^{1 / 2} \tau(c),
$$

where $d(c)$ denotes the divisor function.

Let $J_{v}$ denote the standard J-Bessel function. Let $r(n, Q)$ denote the number of representations of $n$ by the quadratic form $Q$, namely,

$$
r(n, Q)=\sharp\left\{m \in Z^{l}: Q(m)=n\right\} .
$$

If $Q(x)=x_{1}^{2}+x_{2}^{2}+\cdots+x_{l}^{2}$, we denote $r(n, Q):=r_{l}(n)$. Let $A$ be a symmetric positive definite integral matrix associated to $Q$, and let $D=\operatorname{det} A$ denote the discriminant of $A$. Let $G_{Q}(c, a)=\sum_{x \bmod c} e(a Q(x) / c), \delta=(c, D), D=\delta D_{c}$, and $Q^{*}$ be a positive definite integral quadratic form, which is defined in terms of the Smith normal form of $Q^{\dagger}$ (see [14]), and $Q^{\dagger}$ the adjoint form of $Q$.

Then, we have the following Voronoi summation formula [13].

Lemma 1. Let $(c, a)=1$ and $F \in C_{0}^{\infty}\left(R^{+}\right)$be a smooth compact function. We have

$$
\sum_{n=1}^{\infty} r(n, Q) e\left(\frac{a n}{c}\right) F(n)=\frac{(2 \pi)^{m / 2} c^{-m}}{\Gamma(m / 2) \sqrt{D}} G_{Q}(c, a) \Phi\left(\frac{m}{2}\right)+\sum_{n=1}^{\infty} r\left(n, Q^{*}\right) e\left(\frac{-\overline{a D_{c}} n}{c}\right)(\delta n)^{(1-(m / 2)) / 2} G(n),
$$

where

$$
G(n)=\frac{4 \pi D^{(m / 4)-1}}{c^{(m / 2)+1}} G_{Q}(c, a) \int_{0}^{\infty} F(x) x^{((m / 2)-1) / 2} J_{(m / 2)-1}\left(\frac{4 \pi \sqrt{\delta n x}}{c \sqrt{D}}\right) \mathrm{d} x
$$

and $\Phi(s)$ is the Mellin transform of $F(x)$, which is given by

$$
\Phi(s)=\int_{0}^{\infty} F(x) x^{s-1} \mathrm{~d} x .
$$

Remark 1. In our situation, $D=4$, but we still want to compute the dependence of $D$ in our proof. If one can obtain the asymptotic formula for general $r(n, \mathrm{Q})$, then our result can be applied directly to get the analogues for $r(n, Q)$.

For asymptotic expansions of the Bessel functions, we quote the following lemma.

Lemma 2. For $z>0$ large, we have

$$
J_{ \pm v}(z)=\sqrt{\frac{2}{\pi z}}\left\{\cos \left(z \mp \frac{\pi}{2} v-\frac{\pi}{4}\right)-\frac{v^{2}-(1 / 4)}{2 z} \sin \left(z \mp \frac{\pi}{2} v-\frac{\pi}{4}\right)+O\left(z^{-2}\right)\right\} .
$$
[16].

For the mean value of $r(n)$, we have the classical result
(b) If $F^{\prime \prime}(x) \geq v>0$ or $F^{\prime \prime}(x) \leq-v<0$, then

Lemma 3.

$$
\sum_{n \leq x} r(n)=\pi x+O\left(x^{1 / 3}\right) .
$$

We also need the following result [17].

Lemma 4. Let $G(x)$ and $F(x)$ be real functions in $[a, b]$ with $G(x) / F^{\prime}(x)$ being monotonic. Suppose that $|G(x)| \leq M$.

(a) If $F^{\prime}(x) \geq u>0$ or $F^{\prime}(x) \leq-u<0$, then

$$
\int_{a}^{b} G(x) e(F(x)) \mathrm{d} x \ll \frac{M}{u}
$$

$$
\int_{a}^{b} G(x) e(F(x)) \mathrm{d} x \ll \frac{M}{\sqrt{v}} .
$$

\section{Proof of Theorem 1}

In this section, we will finish the proof of Theorem 1. By the formula of the Ramanujan sum

$$
\sum_{d \mid q} \sum_{a=1}^{d} e\left(\frac{a n}{d}\right)= \begin{cases}0, & \text { if } q \nmid n, \\ q, & \text { if } q \mid n,\end{cases}
$$




$$
\sum_{\substack{n \rightarrow X \\ n \equiv l \bmod q}} r(n) e\left(\alpha n^{\beta}\right)=\frac{1}{q} \sum_{c \mid q} \sum_{a \bmod c} * e\left(-\frac{a l}{c}\right) \sum_{n \sim X} r(n) e\left(\frac{a n}{c}\right) e\left(\alpha n^{\beta}\right)
$$

where $\sum^{*}$ means the summation is restricted by $(a, c)=1$.

Let $\Delta>1$, and let $0 \leq \phi(x) \leq 1$ be a $C^{\infty}$ function supported on $[1,2]$, which is identically 1 on $\left[1+\Delta^{-1}, 2-\Delta^{-1}\right]$ and satisfies $\phi^{(r)}(x) \ll \Delta^{r}$ for $r \geq 0$. Using the bound in Lemma 3, we get

$$
\sum_{n \sim X} r(n) e\left(\frac{a n}{c}\right) e\left(\alpha n^{\beta}\right)=\sum_{n=1}^{\infty} r(n) e\left(\frac{a n}{c}\right) W(n)+R_{1}(N, X, \Delta),
$$

where

$$
\begin{aligned}
W(x) & =\phi\left(\frac{x}{X}\right) e\left(\alpha x^{\beta}\right) \\
R_{1}(N, X, \Delta) & \ll \sum_{X<n \leq X+(X / \Delta)}|r(n)| \\
& \ll\left(X+\frac{X}{\Delta}\right)+\left(X+\frac{X}{\Delta}\right)^{(1 / 3)}-X-X^{(1 / 3)} \\
& \ll\left(\frac{X}{\Delta}\right)^{1+\varepsilon} .
\end{aligned}
$$
have

Applying Lemma 1 with $m=2$ and $F(n)=W(n)$, we

$$
\begin{aligned}
\sum_{n=1}^{\infty} r(n) e\left(\frac{a n}{c}\right) W(n)= & \frac{2 \pi}{c^{2} \sqrt{D}} G_{Q}(c, a) \int_{0}^{\infty} W(x) \mathrm{d} x \\
& +\sum_{n=1}^{\infty} r\left(n, Q^{*}\right) e\left(\frac{-\overline{a D_{c}} n}{c}\right) G(n),
\end{aligned}
$$

where

$$
G(n)=\frac{4 \pi}{c^{2} \sqrt{D}} G_{Q}(c, a) \int_{0}^{\infty} W(x) J_{0}\left(\frac{4 \pi \sqrt{\delta n x}}{c \sqrt{D}}\right) \mathrm{d} x .
$$

For the first term, changing variables $x=X t$ and applying Lemma 4(a), we get

$$
\int_{0}^{\infty} W(x) \mathrm{d} x=X \int_{1}^{2} \phi(t) e\left(\alpha(X t)^{\beta}\right) \mathrm{d} t \ll X\left(|\alpha| \beta X^{\beta}\right)^{-1} .
$$

By [16], we have $G_{Q}(c, a) \ll c$. Thus, the contribution of the first term to (22) is

$$
\begin{aligned}
& \frac{1}{q} \sum_{c \mid q} \sum_{a \bmod c} * e\left(-\frac{a l}{c}\right) \frac{2 \pi}{c^{2} \sqrt{D}} G_{Q}(c, a) X\left(|\alpha| \beta X^{\beta}\right)^{-1} \\
& \ll \frac{1}{q} \sum_{c \mid q} \sum_{a \bmod c} * e\left(-\frac{a l}{c}\right) \frac{1}{c} X\left(|\alpha| \beta X^{\beta}\right)^{-1} \\
& \ll q^{-1+\varepsilon} X\left(|\alpha| \beta X^{\beta}\right)^{-1} .
\end{aligned}
$$

Next, we turn to estimate the contribution from the term involving $G(n)$. Using Lemma 2, we have

$$
J_{0}(z)=\frac{1-i}{2 \sqrt{\pi z}}\left(e^{i z}+i e^{-i z}\right)-\frac{1+i}{16 \sqrt{\pi z^{3}}}\left(e^{i z}-i e^{-i z}\right)+O\left(z^{-5 / 2}\right) \text {. }
$$

Taking $z=(4 \pi \sqrt{\delta n x}) / c \sqrt{D}$, we obtain

$$
\begin{aligned}
J_{0}\left(\frac{4 \pi \sqrt{\delta x y}}{c \sqrt{D}}\right)= & \frac{(1-i) c^{1 / 2} D^{1 / 4}}{4 \pi \delta^{1 / 4}}(x y)^{-1 / 4}\left(e\left(\frac{2 \sqrt{\delta x y}}{c \sqrt{D}}\right)+i e\left(-\frac{2 \sqrt{\delta x y}}{c \sqrt{D}}\right)\right)-\frac{(1+i) c^{3 / 2} D^{3 / 4}}{128 \pi^{2} \delta^{3 / 4}}(x y)^{-3 / 4}\left(e\left(\frac{2 \sqrt{\delta x y}}{c \sqrt{D}}\right)\right. \\
& \left.-i e\left(-\frac{2 \sqrt{\delta x y}}{c \sqrt{D}}\right)\right)+O\left(c^{5 / 2}(x y)^{-5 / 4}\right) .
\end{aligned}
$$

Putting this in $G(y)$, we get 


$$
\begin{aligned}
& G(y)=\frac{(1-i)}{c^{3 / 2} D^{1 / 4} \delta^{1 / 4}} G_{Q}(c, a) \int_{0}^{\infty}(x y)^{-1 / 4} \phi\left(\frac{x}{X}\right) e\left(\alpha x^{\beta}\right)\left(e\left(\frac{2 \sqrt{\delta x y}}{c \sqrt{D}}\right)+i e\left(-\frac{2 \sqrt{\delta x y}}{c \sqrt{D}}\right)\right) \mathrm{d} x \\
& -\frac{(1+i) D^{1 / 4}}{32 \pi c^{1 / 2} \delta^{3 / 4}} G_{Q}(c, a) \int_{0}^{\infty}(x y)^{-3 / 4} \phi\left(\frac{x}{X}\right) e\left(\alpha x^{\beta}\right)\left(e\left(\frac{2 \sqrt{\delta x y}}{c \sqrt{D}}\right)-i e\left(-\frac{2 \sqrt{\delta x y}}{c \sqrt{D}}\right)\right) \mathrm{d} x \\
& +O\left(c^{3 / 2} \int_{0}^{\infty}(x y)^{-5 / 4} \phi\left(\frac{x}{X}\right) \mathrm{d} x\right) .
\end{aligned}
$$

Changing variable $x=X t^{2}$, we obtain

$$
\begin{aligned}
G(y)= & \frac{2(1-i)}{c^{3 / 2} D^{1 / 4} \delta^{1 / 4}} G_{Q}(c, a) X^{3 / 4} y^{-1 / 4} \int_{0}^{\infty} t^{-1 / 2} \phi\left(t^{2}\right) e\left(\alpha X^{\beta} t^{2 \beta}\right)\left(e\left(\frac{2 \sqrt{\delta X y}}{c \sqrt{D}} t\right)+i e\left(-\frac{2 \sqrt{\delta X y}}{c \sqrt{D}} t\right)\right) \mathrm{d} t \\
& -\frac{(1+i) D^{1 / 4}}{16 \pi c^{1 / 2} \delta^{3 / 4}} G_{Q}(c, a) X^{1 / 4} y^{-3 / 4} \int_{0}^{\infty} t^{-1 / 2} \phi\left(t^{2}\right) e\left(\alpha X^{\beta} t^{2 \beta}\right)\left(e\left(\frac{2 \sqrt{\delta X y}}{c \sqrt{D}} t\right)-i e\left(-\frac{2 \sqrt{\delta X y}}{c \sqrt{D}} t\right)\right) \mathrm{d} t \\
& +O\left(c^{3 / 2} \int_{0}^{\infty}(x y)^{-5 / 4} \phi\left(\frac{x}{X}\right) \mathrm{d} x\right) \\
= & G_{1}(y)+O\left(c^{3 / 2} X^{-1 / 4} y^{-5 / 4}\right),
\end{aligned}
$$

where

$$
\begin{aligned}
G_{1}(y)= & a_{1} c^{-3 / 2} X^{3 / 4} y^{-1 / 4} G_{Q}(c, a)\left(P_{+}\left(\frac{2 \sqrt{\delta X y}}{c \sqrt{D}}\right)+i P_{+}\left(-\frac{2 \sqrt{\delta X y}}{c \sqrt{D}}\right)\right) \\
& +a_{2} c^{-1 / 2} X^{1 / 4} y^{-3 / 4} G_{Q}(c, a)\left(P_{-}\left(\frac{2 \sqrt{\delta X y}}{c \sqrt{D}}\right)-i P_{-}\left(-\frac{2 \sqrt{\delta X y}}{c \sqrt{D}}\right)\right)
\end{aligned}
$$

with

$$
\begin{aligned}
a_{1} & =\frac{2(1-i)}{D^{1 / 4} \delta^{1 / 4}}, \\
a_{2} & =-\frac{(1+i) D^{1 / 4}}{16 \pi \delta^{3 / 4}}, \\
P_{ \pm}(w) & =\int_{0}^{\infty} t^{ \pm 1 / 2} \phi\left(t^{2}\right) e\left(\alpha X^{\beta} t^{2 \beta}+w t\right) \mathrm{d} t .
\end{aligned}
$$

The O-term contributes

$$
\begin{aligned}
\frac{1}{q} \sum_{c \mid q} & \sum_{n=1}^{\infty} r\left(n, Q^{*}\right)\left|S\left(-l,-\overline{D_{c}} n ; c\right)\right| c^{3 / 2} X^{-1 / 4} n^{-5 / 4} \\
\ll & q^{1+\varepsilon} X^{-(1 / 4)+\varepsilon} .
\end{aligned}
$$

The integral $P_{ \pm}(w)$ defined in (36) was studied by Ren and Ye [7], Sun and Wu [11], and Yan [12]. Here, we follow their steps and choose the parameters with a few differences to get the $q$-aspect saving.
Apply the method given in [7], we obtain

$$
P_{ \pm}(w) \ll \frac{\Delta^{h-1}}{\left(\max \left\{|\alpha| \beta X^{\beta},|w|\right\}\right)^{h}}, \quad h=1,2 .
$$

We will take $w= \pm(2 \sqrt{\delta y X}) /(c \sqrt{D})$ here. Then, $|w| \in\left[(1 / 2)|\alpha| \beta X^{\beta}, 4|\alpha| \beta X^{\beta}\right]$ implies $y \in I$, where

$$
I=\left[\frac{1}{16} \delta^{-1} c^{2} D(|\alpha| \beta)^{2} X^{2 \beta-1}, 4 \delta^{-1} c^{2} D(|\alpha| \beta)^{2} X^{2 \beta-1}\right] .
$$

Thus, for $y=n \notin I$, one obtains by (34) and (38) that $G_{1}(y) \ll\left(c^{-3 / 2} X^{3 / 4} y^{-1 / 4}+c^{-1 / 2} X^{1 / 4} y^{-3 / 4}\right) G_{Q}(c, a) R_{2}(X, y)$,

where

$$
R_{2}(X, y) \ll \frac{\Delta^{h-1}}{(\sqrt{X y} / c)^{h}}, \quad h=1,2 .
$$

Thus, the contrition from $G_{1}(n)$ with $n \notin I$ is 


$$
\begin{aligned}
& \frac{1}{q} \sum_{c \mid q} \sum_{a \bmod c}{ }^{*} e\left(-\frac{a l}{c}\right) \sum_{n \notin I} r\left(n, Q^{*}\right) e\left(\frac{-\overline{a D_{c}} n}{c}\right) G_{1}(n) \\
& \ll \frac{1}{q} \sum_{c \mid q} \sum_{n=1}^{\infty} r\left(n, Q^{*}\right)\left|S\left(-l,-\overline{D_{c}} n ; c\right)\right|\left(c^{-1 / 2} X^{3 / 4} n^{-1 / 4}+c^{1 / 2} X^{1 / 4} n^{-3 / 4}\right) R_{2}(X, n) .
\end{aligned}
$$

Let

$$
Y=\Delta^{2} c^{2} X^{-1}
$$

$$
h= \begin{cases}1, & \text { if } n \leq Y, \\ 2, & \text { if } n>Y,\end{cases}
$$

Applying (41) with

we get

$$
\begin{aligned}
& \ll \frac{1}{q} \sum_{c \mid q} \sum_{n \leq Y} r\left(n, Q^{*}\right)\left|S\left(-l,-\overline{D_{c}} n ; c\right)\right|\left(c^{-1 / 2} X^{3 / 4} n^{-1 / 4}+c^{1 / 2} X^{1 / 4} n^{-3 / 4}\right)\left(c X^{-1 / 2} n^{-1 / 2}\right) \\
& \quad+\frac{1}{q} \sum_{c \mid q} \sum_{n>Y} r\left(n, Q^{*}\right)\left|S\left(-l,-\overline{D_{c}} n ; c\right)\right|\left(c^{-1 / 2} X^{3 / 4} n^{-1 / 4}+c^{1 / 2} X^{1 / 4} n^{-3 / 4}\right)\left(c^{2} \Delta X^{-1} n^{-1}\right) \\
& =: \sum_{1}+\sum_{2} .
\end{aligned}
$$

By the bound $\sum_{n \leq x} r(n)=\pi x+O\left(x^{1 / 3}\right)$, we obtain

$$
\begin{aligned}
\sum_{1}= & \frac{1}{q} \sum_{c \mid q} \sum_{n \leq Y} r\left(n, Q^{*}\right)\left|S\left(-l,-\overline{D_{c}} n ; c\right)\right|\left(c^{1 / 2} X^{1 / 4} n^{-3 / 4}+c^{3 / 2} X^{-1 / 4} n^{-5 / 4}\right) \\
& \ll \frac{1}{q} \sum_{c \mid q} d(c) c X^{1 / 4} \sum_{n \leq Y} r\left(n, Q^{*}\right)\left(l, \overline{D_{c}} n ; c\right)^{1 / 2} n^{-3 / 4} \\
& +\frac{1}{q} \sum_{c \mid q} d(c) c^{2} X^{-1 / 4} \sum_{n \leq Y} r\left(n, Q^{*}\right)\left(l, \overline{D_{c}} n ; c\right)^{1 / 2} n^{-5 / 4} \\
& \ll q^{(1 / 2)+\varepsilon} \Delta^{(1 / 2)+\varepsilon} .
\end{aligned}
$$
we get

Similarly, using the bound $\sum_{n=1}^{\infty}\left(\left|r\left(n, Q^{*}\right)\right|^{2} / n^{1+\varepsilon}\right) \ll 1$, 


$$
\begin{aligned}
& \sum_{2}= \frac{1}{q} \sum_{c \mid q} \sum_{n>Y} r\left(n, Q^{*}\right)\left|S\left(-l,-\overline{D_{c}} n ; c\right)\right| \Delta\left(c^{3 / 2} X^{-1 / 4} n^{-5 / 4}+c^{5 / 2} X^{-3 / 4} n^{-7 / 4}\right) \\
& \ll \frac{1}{q} \Delta \sum_{c \mid q} d(c) c^{2+\varepsilon} X^{-1 / 4} \sum_{n>Y} r\left(n, Q^{*}\right)\left(l, \overline{D_{c}} n ; c\right)^{1 / 2} n^{-5 / 4} \\
&+\frac{1}{q} \Delta \sum_{c \mid q} d(c) c^{3+\varepsilon} X^{-3 / 4} \sum_{n>Y} r\left(n, Q^{*}\right)\left(l, \overline{D_{c}} n ; c\right)^{1 / 2} n^{-7 / 4} \\
& \ll \frac{1}{q} \Delta \sum_{c \mid q} c^{2+\varepsilon} X^{-1 / 4}\left(\sum_{n>Y} \frac{\left|r\left(n, Q^{*}\right)\right|^{2}}{n^{1+\varepsilon}}\right)^{1 / 2}\left(\sum_{n>Y} n^{-3 / 2}\right)^{1 / 2} \\
&+\frac{1}{q} \Delta \sum_{c \mid q} c^{3+\varepsilon} X^{-3 / 4}\left(\sum_{n>Y} \frac{\left|r\left(n, Q^{*}\right)\right|^{2}}{n^{1+\varepsilon}}\right)^{1 / 2}\left(\sum_{n>Y} n^{-5 / 2}\right)^{1 / 2} \\
& \ll \frac{1}{q} \Delta \sum_{c \mid q} c^{2+\varepsilon} X^{-1 / 4} Y^{-(1 / 4)+\varepsilon}+\frac{1}{q} \Delta \sum_{c \mid q} c^{3+\varepsilon} X^{-3 / 4} Y^{-(3 / 4)+\varepsilon} \\
& \ll q(1 / 2)+\varepsilon \\
& \Delta^{(1 / 2)+\varepsilon} .
\end{aligned}
$$

Finally, we obtain the contribution of (45) as

$$
\ll q^{(1 / 2)+\varepsilon} \Delta^{(1 / 2)+\varepsilon} \text {. }
$$

If $|\alpha| \beta q X^{\beta}<(1 / 2) \sqrt{\delta X / D}$, then $I \cap Z^{+}=\varnothing$, by (25), (29), (37), and (48), and choosing

$$
\Delta=X^{2 / 3} q^{-1 / 3}
$$

we get

$$
\sum_{n \sim X n \equiv l \bmod q} r(n) e\left(\alpha n^{\beta}\right) \ll q^{(1 / 3)+\varepsilon} X\left(|\alpha| \beta X^{\beta}\right)^{-1} .
$$

Next, we will handle the situation that $y=n \in I$. By the trivial estimate $\int_{1}^{\sqrt{2}} t^{ \pm 1 / 2} \phi\left(t^{2}\right) e(f(t)) \mathrm{d} t \ll 1$ and putting it into (22), we get the second term in the right-hand side of (34) which contributes

$$
\ll \frac{1}{q} \sum_{c \mid q} \sum_{n \in I} r\left(n, Q^{*}\right)\left|S\left(-l,-\overline{D_{c}} n ; c\right)\right| c^{-1 / 2} X^{1 / 4} n^{-3 / 4} G_{Q}(c, a)
$$$$
\ll q^{(1 / 2)+\varepsilon}\left(|\alpha| \beta X^{\beta}\right)^{(1 / 2)+\varepsilon}
$$

Applying (38) to bound the terms with $\alpha w>0$, we have

$$
P_{+}\left(\frac{2 \sqrt{\delta X y}}{c \sqrt{D}}\right)+i P_{+}\left(-\frac{2 \sqrt{\delta X y}}{c \sqrt{D}}\right)=\varepsilon_{\alpha} \int_{0}^{\infty} t^{1 / 2} \phi\left(t^{2}\right) e\left(f_{1}(t)\right) \mathrm{d} t+O\left(R_{2}(X, y, c)\right)
$$

where

$$
\begin{aligned}
\epsilon_{\alpha} & = \begin{cases}i, & \alpha>0, \\
1, & \alpha<0,\end{cases} \\
f_{1}(t) & =f_{1}(t, y)=\operatorname{sgn}(\alpha)\left(|\alpha| X^{\beta} t^{2 \beta}-\frac{2 \sqrt{\delta X y}}{c \sqrt{D}} t\right) .
\end{aligned}
$$

$$
\varepsilon_{\alpha} \frac{a_{1}}{q} \sum_{c \mid q} c^{-3 / 2} X^{3 / 4} \sum_{n \in I} r\left(n, Q^{*}\right)\left|S\left(-l,-\overline{D_{c}} n ; c\right)\right| n^{-1 / 4} G_{Q}(c, a) \int_{1}^{\sqrt{2}} t^{1 / 2} \phi\left(t^{2}\right) e\left(f_{1}(t, n)\right) \mathrm{d} t .
$$


We distinguish two cases according to $\beta=1 / 2$ or not. If $\beta \neq 1 / 2$, we have

$$
f_{1}^{\prime \prime}(t)=\operatorname{sgn}(\alpha)|\alpha|(2 \beta)(2 \beta-1) X^{\beta} t^{2 \beta-2} \gg|\alpha| \beta|2 \beta-1| X^{\beta}, \quad \text { for } t \in[1, \sqrt{2}] .
$$

By integration by parts and [17], we have

$$
\int_{1}^{\sqrt{2}} t^{1 / 2} \phi\left(t^{2}\right) e\left(f_{1}(t, n)\right) \mathrm{d} t \ll \max _{1 \leq t \leq \sqrt{2}}\left|\int_{1}^{t} e\left(f_{1}(u)\right) \mathrm{d} u\right| \ll\left(|\alpha| \beta|2 \beta-1| X^{\beta}\right)^{-1 / 2}
$$

Hence, (55) is

$$
\begin{aligned}
& \left.\ll \frac{1}{q} \sum_{c \mid q} c^{-3 / 2} X^{3 / 4} \sum_{n \in I} r\left(n, Q^{*}\right)\left|S\left(-l,-\overline{D_{c}} n ; c\right)\right| n^{-1 / 4} G_{Q}(c, a)|\alpha| \beta|2 \beta-1| X^{\beta}\right)^{-1 / 2} \\
& \ll q^{(1 / 2)+\varepsilon}|2 \beta-1|^{-(1 / 2)}\left(|\alpha| \beta X^{\beta}\right)^{1+\varepsilon} .
\end{aligned}
$$

If $\beta=1 / 2$, let

$$
\left|A\left(H_{r}\right)\right| \leq H_{r} c|\alpha| X^{-1 / 2} .
$$

$H_{r}=2^{-r} c|\alpha| X^{1 / 2}, \quad 1 \leq r \leq r_{0}=\left[\log _{2}\left(c|\alpha| X^{1 / 2}\right)\right]+1$,

Moreover, for $n \in A\left(H_{r}\right)$, we have

$$
\left|f_{1}^{\prime}(t)\right|=|| \alpha\left|-\frac{2 \sqrt{\delta n}}{c \sqrt{D}}\right| X^{1 / 2}>H_{r} c^{-1}
$$

and write

$$
A\left(H_{r}\right)=\left\{n: H_{r} X^{-1 / 2}<|c| \alpha\left|-2 \sqrt{\frac{\delta n}{D}}\right| \leq 2 H_{r} X^{-1 / 2}\right\}
$$

Then, we obtain

$$
\int_{1}^{\sqrt{2}} t^{1 / 2} \phi\left(t^{2}\right) e\left(f_{1}(t)\right) \mathrm{d} t \ll H_{r}^{-1} c
$$

Then,

Hence, in this situation, (55) contributes

$$
\begin{aligned}
& \ll \frac{1}{q} \sum_{c \mid q} c^{-1 / 2} X^{(3 / 4)+\varepsilon} \sum_{r=1}^{r_{0}} H_{r}^{-1} \sum_{n \in A\left(H_{r}\right)} r\left(n, Q^{*}\right)\left|S\left(-l,-\overline{D_{c}} n ; c\right)\right| n^{-1 / 4} G_{Q}(c, a) \\
& \ll \frac{1}{q} \sum_{c \mid q} c^{-1 / 2} X^{(3 / 4)+\varepsilon} \sum_{r=1}^{r_{0}} H_{r}^{-1}\left(|\alpha|^{2} c^{2}\right)^{-(1 / 4)+\varepsilon} c^{(3 / 2)+\varepsilon}\left|A\left(H_{r}\right)\right| \\
& \ll q^{(1 / 2)+\varepsilon} X^{(1 / 4)+\varepsilon}|\alpha|^{(1 / 2)+\varepsilon} .
\end{aligned}
$$

It remains to estimate

$$
\varepsilon_{\alpha} \frac{a_{1}}{q} \sum_{c \mid q} c^{-3 / 2} X^{3 / 4} \sum_{n \in I \cap I_{0}} r\left(n, Q^{*}\right)\left|S\left(-l,-\overline{D_{c}} n ; c\right)\right| n^{-1 / 4} G_{Q}(c, a) \int_{1}^{\sqrt{2}} t^{1 / 2} \phi\left(t^{2}\right) e\left(f_{1}(t, n)\right) \mathrm{d} t
$$

where 


$$
I_{0}=\left\{n:|c| \alpha\left|-2 \sqrt{\frac{\delta n}{D}}\right| \leq X^{-1 / 2}\right\}
$$

Note that

$$
\left|\left\{n:|c| \alpha\left|-2 \sqrt{\frac{\delta n}{D}}\right| \leq X^{-1 / 2}\right\}\right| \leq c|\alpha| X^{-1 / 2} .
$$

If $|\alpha| \leq(1 / q)$, then $I_{0}=\varnothing$, and we have (65) which vanishes. And if $|\alpha| \geq(\sqrt{X} / q)$, then by trivial estimate, (65) contributes

$$
\begin{aligned}
& \frac{1}{q} \sum_{c \mid q} c^{-3 / 2} X^{3 / 4} \sum_{|c| \alpha|-2 \sqrt{(\delta n / D)}| \leq X^{-1 / 2}} r\left(n, Q^{*}\right)\left|S\left(-l,-\overline{D_{c}} n ; c\right)\right|^{n-1 / 4} G_{Q}(c, a) \\
& \quad \ll \frac{1}{q} \sum_{c \mid q} c^{1 / 2} X^{3 / 4}\left|I_{0}\right|\left(c^{2}|\alpha|^{2}\right)^{-(1 / 4)+\varepsilon} \\
& \ll q^{\varepsilon} X^{(1 / 4)+\varepsilon}|\alpha|^{(1 / 2)+\varepsilon} .
\end{aligned}
$$

If $(1 / q) \leq|\alpha| \leq(\sqrt{X} / q)$, then there is at most one integer for every $c \mid q$, say $n=n_{c}$. Hence, (55) becomes satisfying

$$
|c| \alpha\left|-2 \sqrt{\frac{\delta n}{D}}\right| \leq X^{-1 / 2}
$$

$$
\begin{aligned}
& \varepsilon_{\alpha} \frac{a_{1}}{q} \sum_{c \mid q} \delta_{c} c^{-3 / 2} X^{3 / 4} r\left(n_{c}, Q^{*}\right)\left|S\left(-l,-\overline{D_{c}} n_{c} ; c\right)\right| n_{c}^{-1 / 4} G_{Q}(c, a) \int_{1}^{\sqrt{2}} t^{1 / 2} \phi\left(t^{2}\right) e\left(f_{1}\left(t, n_{c}\right)\right) \mathrm{d} t \\
& \quad=\frac{\varepsilon_{\alpha} a_{1}}{2 q} \sum_{c \mid q} \delta_{c} c^{-3 / 2} X^{3 / 4} r\left(n_{c}, Q^{*}\right)\left|S\left(-l,-\overline{D_{c}} n_{c} ; c\right)\right| n_{c}^{-1 / 4} G_{Q}(c, a) \int_{1}^{2} u^{-1 / 4} e\left(f_{1}\left(u^{1 / 2}, n_{c}\right)\right) \mathrm{d} u+O\left(c^{-(1 / 2)+\varepsilon} \Delta^{-1} X^{3 / 4} r\left(n_{c}, Q^{*}\right) n_{c}^{-1 / 4}\right) \\
& \quad=\frac{1}{q} \sum_{c \mid q} \varepsilon\left(a, n_{c}\right) c^{-3 / 2} X^{3 / 4} r\left(n_{c}, Q^{*}\right)\left|S\left(-l,-\overline{D_{c}} n_{c} ; c\right)\right| n_{c}^{-1 / 4} G_{Q}(c, a)+O(1)
\end{aligned}
$$

where

$$
\varepsilon\left(a, n_{c}\right)=\frac{\delta_{c} \varepsilon_{\alpha} a_{1}}{2} \int_{1}^{2} u^{-1 / 4} e\left(\operatorname{sgn}(\alpha)\left(|\alpha|-\frac{2 \sqrt{\delta n_{c}}}{c \sqrt{D}}\right) \sqrt{X u}\right) \mathrm{d} u
$$

$$
\frac{1}{q} \sum_{c \mid q} \varepsilon\left(a, n_{c}\right) c^{-3 / 2} X^{3 / 4} r\left(n_{c}, Q^{*}\right)\left|S\left(-l,-\overline{D_{c}} n_{c} ; c\right)\right| n_{c}^{-1 / 4} G_{Q}(c, a)
$$

and $\delta_{c}=1$ or 0 according to if there exists such $n_{c}$ or not. Recall

$$
\begin{aligned}
& a_{1}=\frac{2(1-i)}{D^{1 / 4} \delta^{1 / 4}}, \\
& \varepsilon_{\alpha}= \begin{cases}i, & \alpha>0, \\
1, & \alpha<0 .\end{cases}
\end{aligned}
$$

When $\beta=1 / 2$ and $(1 / q) \leq|\alpha| \leq(\sqrt{X} / q)$ and applying (64) and (68), we derive that (55) equals to

This proves Theorem 1(iii).

In particular, if $|\alpha|=(2 / q) \sqrt{\delta k / D}$ with $1 \leq k \leq(D X / 4 \delta)$, then for $c \mid q, c \neq q$, we have

$$
|c| \alpha\left|-2 \sqrt{\frac{\delta k}{D} \mid}\right|=2 \sqrt{\frac{\delta k}{D}}\left|\frac{c}{q}-1\right| \geq \frac{2}{q} \sqrt{\frac{\delta k}{D}} \geq 2 \sqrt{\frac{\delta k}{D X}} \geq 1 .
$$

Thus, $\delta_{c}=0$ for $c \neq q$ in this situation. Hence, (73) becomes 


$$
\begin{aligned}
\sum_{n \sim X n \equiv l \bmod q} r(n) e\left( \pm \frac{2}{q} \sqrt{\frac{\delta k n}{D}}\right)= & \frac{4}{3}\left(2^{3 / 4}-1\right) D^{-1 / 4} \delta^{-1 / 4}(1 \pm i)\left|S\left(-l,-\overline{D_{c}} k ; q\right)\right| q^{-5 / 2} X^{3 / 4} r\left(k, Q^{*}\right) k^{-1 / 4} G_{Q}(q, a) \\
& +O\left(k^{-1 / 4} X^{1 / 4}(q X k)^{\varepsilon}\right) .
\end{aligned}
$$

This proves Theorem 1(iv).

\section{Data Availability}

The data used to support the findings of this study are included within the article.

\section{Conflicts of Interest}

The authors declare that they have no conflicts of interest.

\section{Acknowledgments}

Xiaofei Yan is supported by National Natural Science Foundation of China (Grant No. 11801327) and Natural Science Foundation of Shandong Province (Grant No. ZR201709280100).

\section{References}

[1] I. M. Vinogradov, "Estimate of a prime-number trigonometric sum," Izvestiya Rossiiskoi Akademii Nauk. Seriya Matematicheskaya, vol. 1, pp. 157-164, 1959.

[2] H. Iwaniec, W. Luo, and P. Sarnak, "Low lying zeros of families of L-functions," Publications mathématiques de l'IHÉS, vol. 91, no. 1, pp. 55-131, 2000.

[3] H. Lao and A. Sankaranarayanan, "The distribution of fourier coefficients of cusp forms over sparse sequences," Acta Arithmetica, vol. 163, no. 2, pp. 101-110, 2014.

[4] H. Lao, M. McKee, and Y. Ye, "Asymptotics for cuspidal representations by functoriality from GL(2)," Journal of Number Theory, vol. 164, pp. 323-342, 2016.

[5] H.-F. Liu, "Mean value estimates of the coefficients of product L-functions," Acta Mathematica Hungarica, vol. 156, no. 1, pp. 102-111, 2018.

[6] S. D. Miller and W. Schmid, "The highly oscillatory behavior of automorphic distributions for SL(2)," Letters in Mathematical Physics, vol. 69, no. 1-3, pp. 265-286, 2004.

[7] X. Ren and Y. Ye, "Resonance between automorphic forms and exponential functions," Science China Mathematics, vol. 53, pp. 2463-2472, 2000.

[8] Q. Sun and D. Zhang, "Sums of the triple divisor function over values of a ternary quadratic form," Journal of Number Theory, vol. 168, pp. 215-246, 2016.

[9] D. Zhang and Y. Wang, "Ternary quadratic form with prime variables attached to Fourier coefficients of primitive holomorphic cusp form," Journal of Number Theory, vol. 176, pp. 211-225, 2017.

[10] D. Zhang and Y. Wang, "Higher-power moments of Fourier coefficients of holomorphic cusp forms for the congruence subgroup \$ \$vargamma _0 (N)\$ $\$ 0(\mathrm{~N})$," The Ramanujan Journal, vol. 47, no. 3, pp. 685-700, 2018.

[11] Q. Sun and Y. Wu, "Exponential sums involving Maass forms," Frontiers of Mathematics in China, vol. 9, no. 6, pp. 1349-1366, 2014.
[12] X. Yan, "On some exponential sums involving maass forms over arithmetic progressions," Journal of Number Theory, vol. 160, pp. 44-59, 2016.

[13] G. Hu, Y. Jiang, and G. Lü, "The fourier coefficients of $\Theta$-series in arithmetic progressions," Mathematika, vol. 66, no. 1, pp. 39-55, 2020.

[14] T. Callahan and R. A. Smith, " $\$ \mathrm{~L} \$$-functions of a quadratic form," Transactions of the American Mathematical Society, vol. 217 , p. $297,1976$.

[15] I. S. Gradshteyn and I. M. Ryzhik, Table of Integrals, Series and Products, Academic Press, Cambridge, MA, USA, 6th edition, 2000.

[16] H. Iwaniec and Kowalski, Analytic Number Theory-American Mathematical Society, Colloquium Publications, Providence, RI, USA, 2004.

[17] E. C. Titchmarsh, The Theory of the Riemann Zeta-Function, Oxford University Press, Oxford, England, UK, 2 edition, 2014. 